\title{
A review of the book titled "Getting Past Your Past" written by Francine Shapiro
}

"Getting Past Your Past: Take Control of Your Life with Self-Help Techniques" is the latest publication by Francine Shapiro, an American psychologist who created and developed Eye Movement Desensitization and Reprocessing (EMDR) therapy.

Eye Movement Desensitization and Reprocessing stands for Eye Movement Desensitisation and Reprocessing. It is a fairly new therapeutic approach aimed at combating traumatic memories and experiences. Practiced by ca. 70,000 clinicians worldwide, and with more than 20 million patients responding positively to the treatment, EMDR has been gaining popularity as an efficient method of alleviating trauma and related disorders, which prevent good quality of life.

In her book, Francine Shapiro presents and addresses a variety of issues, such as who we really are, how our mind, brain, and body are connected, and how traumatic memories and experiences may influence our current self and condition. By describing the examples of actual clinical cases, she helps readers understand how problematic behaviours, feelings, and memories have an adverse impact upon present actions of individuals.

The chapters of the book are structured to provide an outlook on specific matters related with trauma and to demonstrate how EMDR therapy addresses it, while also presenting stories of patients who have successfully overcome their trauma.

One of the problems primarily elaborated by Shapiro is post-traumatic stress disorder (PTSD), which involves extreme emotional distress from significant trauma such as accidents, physical or sexual abuse, war combat, or natural disasters.

When people are not able to process traumatic memories or experiences, they are likely to react in ways that may have made sense in the past, but are no longer helpful in the present. This is one of the key issues described in the first chapter of the book, titled: "Running on Automatic". The majority of experienced problems stem from the past, and while our reactions to them may seem irrational in the present, it does not mean there is no reason for it. The memories are stored in the brain and may emerge without our control. These memories can subsequently interfere with our present life. The book provides guidance to trace current difficulties to past unprocessed memories that are the basis for current responses. Shapiro presents a number of example stories, through which she demonstrates how traumatic events from the past build a network in the brain and impact present life.
The chapter "Mind, Brain, and What Matters" offers a detailed explanation of the way in which the brain blocks the external and internal information when it is in a stressful situation in order to protect it from further damage. It also proves that despite being able to heal itself, the brain is often prevented from it by memories and experiences from the past. In addition, the author provides a description of how certain events in present life can trigger bad memories that have not been processed by the neural networks. Finally, this chapter presents the origins of the EMDR therapy as well as why and how it works.

The following chapter "Is it Climate or the Weather?" deals with issues that may be considered temporary and not as profoundly entrenched, thus referred to as 'weather', as well as the more permanent and recurring ones, considered to be causes for depression ('climate'). As Shapiro points out, the ultimate goal in a therapy is to make changes in the climate rather than the weather to prevent relapses of adverse conditions. This chapter also demonstrates various techniques, such as "Safe or Calm Place", "Breathing Shift Technique", or the "Cartoon Character Technique", which may help us to deal with memories that disturb us. In addition, it presents the notion of Bilateral Stimulation (with such techniques as tapping or Butterfly Hug), extensively used in EMDR therapy. Finally, it familiarises readers with the concept of the "Touchstone Memory" - the earliest unprocessed memory about a traumatic event.

Shapiro continues to demonstrate further techniques and tools in the following section, titled "What's Running Your Show?" Here, the author instructs us how to identify negative cognitions and memories that are responsible for our reactions.

The fifth chapter, "The Hidden Landscape", provides an insight of the types of issues people have to deal with and why there are so many individuals who find it difficult if not impossible to experience satisfaction and well-being in life. The author lists the circumstances and reasons of physical and emotional separation (referring to parent-child relationship) and explains the connection of insecure attachment with anxiety and fear. Like in all chapters, also this one is illustrated with real-life examples.

In the next chapter "I Would if I Could, but I Cannot", Shapiro demonstrates how fear and anxiety of current situations may be warning signals of a past traumatic event and how these otherwise useful emotions can cause irrational reactions to present circum- 
stances. Both fear and anxiety can be an aftermath of various incidents from the past that triggered in patients a lack of safety/vulnerability or lack of power/ control. The chapter also evaluates the previously described tools at our disposal (Safe/Calm Place or Butterfly Hug) that can help individuals reduce the negative consequences of such emotions and reactions.

The relation between the mind and the body are presented in the following chapter, "The Brain, Body, and Mind Connection". It explores conditions often perceived as physical, which may actually be caused by unprocessed memories powerful enough to provoke adverse effects in the brain and body. Shapiro stresses, however, that body reactions can also disturb our peace of mind. In addition, the chapter introduces a self-help method: "Lightstream Technique", useful for people suffering from psychosomatic problems.

The next chapter, "What do You Want from Me?" contains a number of real-life stories presenting problematic issues arising between close people - for instance parents and children, siblings, etc. It focuses on interpersonal relationships between them and how these relationships may be disturbed by unprocessed memories. It also demonstrates how the behaviour of some people may trigger exaggerated or unreasonable reactions in those close to them.

In a particularly moving chapter, titled "A Part of the Whole", Shapiro provides accounts of serious offenders: child molesters, perpetrators of domestic violence, drug addicts, etc. She stresses that, despite the appalling deeds they have committed, the leading concept here is an exploration towards finding understanding rather than guilt.

In the following sections of the book, Shapiro demonstrates how the methods and the techniques introduced earlier can help individuals overcome their phobias and anxiety as well as understand the ways in which they react. In addition, the author stresses that eliminating our suffering is just a first step - she urges that we should expand our potential and capabilities while improving our quality of life and peace of mind. Some advice and information about techniques to achieve it may be found in Chapter 10, titled "From Stressed to Better than Well", in which the author provides valuable instructions, all backed by scientific explanation and real examples. This chapter can be a good source of inspiration for anyone willing to excel in their lives - how to make a transition "from a survivor to thriver", as well as a blessing to all those struggling with stressful life situations.

The final chapter of the book, titled "Bringing it Home", demonstrates what makes people who they are, how they are defined by their cultural and social background, and how the decisions we make influence us and those around us. It also explores how to face unavoidable events in life, such as disease or death, as well as the ways to endure them and move on. This part of the book offers ways of self-exploration that individuals can use to tackle memories preventing them from living joyous and fulfilling lives.

"Getting Past Your Past" is a fascinating book, written in an easy format, yet never straying from scientific accuracy and insight. The author, Francine Shapiro, demonstrates, in an approachable and engaging manner, a variety of issues, ranging from the causes of trauma and poor quality of life, through self-diagnosing and self-exploration techniques, ending with ways to overcome adversities as well as to develop one's own potential. Not a single problem presented in the book is left without a real-life example, making it a captivating publication for readers. Explained in an approachable manner, these techniques can be used even by non-professionals, although Shapiro emphasises that in severe cases the participation of a professional therapist is recommended and required. However, the most prominent aspect of the book seems to be how the distressful conditions experienced by people who have gone through trauma, neglect, or abuse can be, and have been, successfully tackled using EMDR. This fairly new method has proved highly effective in treating trauma and phobic disorders, such as PTSD. Dr Francine Shapiro, the creator of the method, offers a detailed explanation of how it works, and demonstrates that even the most severe cases of abuse, trauma, or other disorders can be treated successfully.

"Getting Past Your Past" is an excellent guide for both professional therapists and for individuals who just want to learn about the EMDR therapy and related issues or who seek to explore their past and present experiences and memories. Some people may consider it a source of knowledge or information; others may be inspired by it to change their lives. In any case, "Getting Past Your Past" by Francine Shapiro is a highly recommended publication not to be missed.

Monika Szpringer Faculty of Medicine and Health Sciences of Jan Kochanowski University in Kielce 